\title{
Autoantibodies to IL-17A may be Correlated with the Severity of Mucocutaneous Candidiasis in APECED Patients
}

\author{
Adrien Katalin Sarkadi • Szilvia Taskó • \\ Gabriella Csorba • Beáta Tóth • Melinda Erdôs • \\ László Maródi
}

Received: 15 May 2013 / Accepted: 3 January 2014 / Published online: 4 February 2014

(C) Springer Science+Business Media New York 2014

\begin{abstract}
The relative roles of various autoantibodies against IL-17-type cytokines in susceptibility to chronic mucocutaneous candidiasis (CMC) in patients with autoimmune polyendocrinopathy-candidiasis-ectodermal dystrophy (APECED) remain poorly defined. The purpose of this longitudinal study was to analyze the relationship between the occurrence of mucocutaneous candidiasis and levels of antiIL-17A, anti-IL-17F and anti-IL-22 autoantibodies. We studied six APECED patients from four families with various disease manifestations. Clinical data were collected during regular follow-up. Anti-endocrine organ antibody levels and clinical chemistry and immunology parameters were determined in routine laboratory assays on freshly isolated serum. Levels of autoantibodies against IL-17A, IL-17F, IL-22, IFN- $\alpha$, IFN- $\omega$ and TNF- $\alpha$, and cytokine release by Candid $\alpha-$ exposed blood cells were determined by ELISA. Mutations were analyzed by sequencing genomic DNA. Four patients carried the germline c.769C $>$ T homozygous nonsense mutation, which results in R257X truncation of the AIRE protein, and two patients from the same family were compound heterozygous for the c.769C $>\mathrm{T} / \mathrm{c} .1344 \mathrm{delC}$ mutation. We found persistently high levels of antibodies against IL-17A in the serum samples of one patient presenting CMC since infancy and low or undetectable anti-IL-17A antibody levels in the sera of five patients with no candidiasis or without severe candidiasis. By contrast, levels of autoantibodies against IL$17 \mathrm{~F}$ and IL-22 were higher in all patients than in healthy controls. Release of IL-17-type cytokines by Candida-
\end{abstract}

Melinda Erdős and László Maródi these authors share last authorship.

A. K. Sarkadi $\cdot$ S. Taskó · G. Csorba · B. Tóth · M. Erdős $(\bowtie)$ · L. Maródi

Department of Infectious and Pediatric Immunology, University of

Debrecen, Medical and Health Science Center, Nagyerdei Krt. 98,

4032 Debrecen, Hungary

e-mail: melinda.erdos@yahoo.com exposed blood mononuclear cells was low or negligible in all patients tested. We suggest that anti-IL-17A antibodies may play an important role in the predisposition to candidiasis of APECED patients. However, the lack of severe CMC in APECED patients with high levels of IL-17F and anti-IL-22 autoantibodies clearly calls into question the role of these antibodies as the principal cause of cutaneous and mucosal candidiasis in at least some APECED patients. These data also suggest that the impaired release of IL-17-type cytokines by blood cells may be an element of the immunopathology of CMC in APECED patients.

Keywords APECED $\cdot$ IL-17 $\cdot$ IL-22 $\cdot$ chronic mucocutaneous candidiasis

\section{Introduction}

Chronic mucocutaneous candidiasis (CMC) associated with hypoparathyroidism was first reported by Thorpe and Handley in 1929 [34]. This condition was commonly referred to as Schmidt's syndrome, after the German pathologist who studied autoimmunity to more than one endocrine organ [9]. Autoimmune polyendocrine syndrome associated with $\mathrm{CMC}$ is a rare autosomal recessive disease and is also referred to as autoimmune polyendocrinopathy-candidiasis-ectodermal dystrophy (APECED) $[10,22]$. APECED is caused by a loss-of-function mutation of the autoimmune regulator gene (AIRE), resulting in the production of a mutant AIRE protein, which allows autoreactive $T$ cells to escape negative selection in the thymus, leading to autoimmunity to various endocrine organs and the production of autoantibodies against selfantigens and various cytokines $[10-12,17-19,25,26]$. The diagnosis of APECED is based on the presence of at least two of three major clinical manifestations: CMC, primary hypoparathyroidism and primary adrenocortical insufficiency 
(Addison's disease) [2, 10, 16, 25]. In most cases, signs and symptoms of the disease appear in infancy or childhood and affected patients typically develop three to seven different disease manifestations. In addition to the major criteria, minor disease components are also common [3, 4, 25, 38]. These signs and the major components of disease often develop in a particular chronological order. CMC commonly begins before the age of 5 years, followed (usually years later) by autoimmune hypoparathyroidism before the age of 10 years and adrenal insufficiency before the age of 15 years $[2,10,20]$.

The earliest and most common clinical manifestation in early childhood is CMC, which is particularly common in patients carrying the Finnish homozygous allele R257X [11, 12]. Oral candidiasis frequently develops during the first year of life, followed by the involvement of the skin and nails, with various degrees of severity $[10,11,17]$. Chronic oral candidiasis may lead to leukoplakia and may increase the risk of squamous epithelial carcinoma. In several cases, carcinoma has been reported in association with mucocutaneous candidiasis of the oropharyngeal cavity and the esophagus, suggesting that persistent mucosal candidiasis may be carcinogenic in the absence of other risk factors [30, 31, 37]. Candidiasis of the nails results in thickening and discoloration, paronychia and nail loss. Candida infection may cause superficial mucous membrane lesions in the esophagus, serious inflammation with strictures, painful swallowing, retrosternal pain and a sensation of food becoming stuck in the chest. Female patients typically develop chronic vulvovaginitis. Hypoparathyroidism generally occurs within 10 years of the onset of candidiasis, but it may manifest at any time in adulthood. Adrenocortical failure generally occurs after the onset of candidiasis and/or hypoparathyroidism, with a peak incidence between the ages of four and 12 years, but hormone levels may fluctuate for years [20]. Adrenal insufficiency is preceded by the production of anti-21-hydroxylase antibodies. Other endocrine signs include ovarian failure, diabetes mellitus type 1, testicular failure, autoimmune thyroiditis and hypophysitis [19, 20, 25, 38]. APECED may be associated with gastrointestinal signs, such as autoimmune gastritis, hepatitis and malabsorption syndromes [10]. Ectodermal dystrophy may include dental enamel hypoplasia, keratopathy, vitiligo, alopecia, nail pitting and tympanic membrane calcifications $[4,38]$. Vitiligo may develop in the first month of life and its extent is highly variable, from isolated spots to coverage of almost the entire body surface [4].

We describe here the longitudinal observation of six APECED patients with various clinical signs and laboratory findings. Our findings suggest that anti-IL-17A, but not antiIL-17F or anti-IL-22 antibodies are the key factors determining susceptibility to candidiasis in APECED patients. We also suggest that there may not be a strong chronological relationship between the production of autoantibodies and the development of clinical signs of APECED. Finally, we present data suggesting that the release of IL-17-type cytokines by the Candida-exposed blood mononuclear cells of APECED patients may be impaired.

\section{Materials and Methods}

\section{Patient 1}

This 10-year-old boy had nail dystrophy, dental enamel hypoplasia and nail pitting from the age of 3.5 years. At the age of 4 years, he presented chickenpox and developed alopecia totalis, involving the scalp, eyelashes and eyebrows, which has persisted ever since (Fig. 1b). Routine laboratory tests showed persistent hyperphosphatemia and high thyroidstimulating hormone (TSH) levels, but serum calcium concentration and liver enzyme activities were normal (Table I). The patient has not had mucosal or skin candidiasis. An adrenal cortex antibody assay showed persistent weak positivity without signs of autoimmune adrenal insufficiency.

\section{Patient 2}

This 7-year-old girl (the sister of Patient 1; Fig. 1a) developed muscle cramps, recurrent upper respiratory tract infections and oral and nail candidiasis caused by Candida albicans at 18 months of age. Calcium and vitamin D supplementation was initiated. At 4 years of age, blood tests showed low serum parathormone (PTH) concentration, hyperphosphatemia, hypergammaglobulinemia and high levels of liver enzymes (Table I). At the age of 5 years, P2 had a low serum cortisol concentration and developed iron deficiency with hypochromic microcytic anemia (Table I). Laboratory and genetic tests excluded celiac disease and DiGeorge syndrome. This patient had organ-specific autoantibodies against liver-kidney microsomal enzymes and the adrenal cortex (Table I). She was treated with anti-fungal agents (borax-glycerin, nystatin, fluconazole), parenteral and oral calcium and vitamin D derivatives from early childhood. At 5 years of age, given the persistence of hypertransaminasemia and treatment-resistant candidiasis, we initiated intravenous immunoglobulin therapy (IVIG), at a dose of $560 \mathrm{mg} / \mathrm{kg}$ monthly. One week after the first session of IVIG treatment, oral candidiasis had improved, and the patient remained symptom-free for 2 weeks. In the following month, before IVIG treatment, laboratory tests showed a decrease in liver enzyme levels and a normal serum calcium concentration. One year after the first IVIG infusion, the patient presented with fever once or twice per week $\left(\mathrm{T}_{\max }\right.$ : $39.3^{\circ} \mathrm{C}$ ), followed by an urticarial rash on her arms and legs. Symptoms ceased spontaneously after 12 to $24 \mathrm{~h}$. Laboratory tests showed high levels of liver enzymes (GOT $78 \mathrm{U} / 1$, GPT $54 \mathrm{U} / \mathrm{l})$ and C-reactive protein $(16.4 \mathrm{mg} / \mathrm{l}$; normal: $<5 \mathrm{mg} / \mathrm{l})$, a high white blood cell count (12.49 G/1; normal range: $4.5-$ 
a

Control

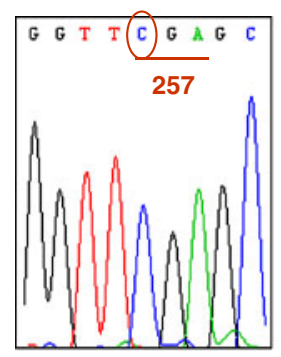

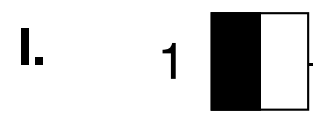
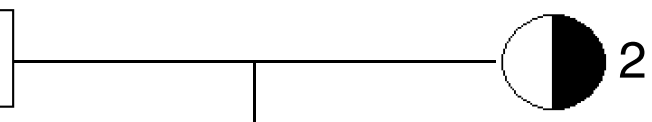

II. 1

P1

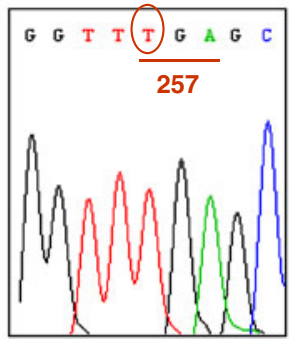

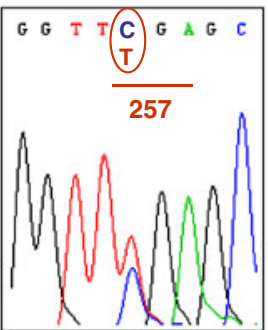
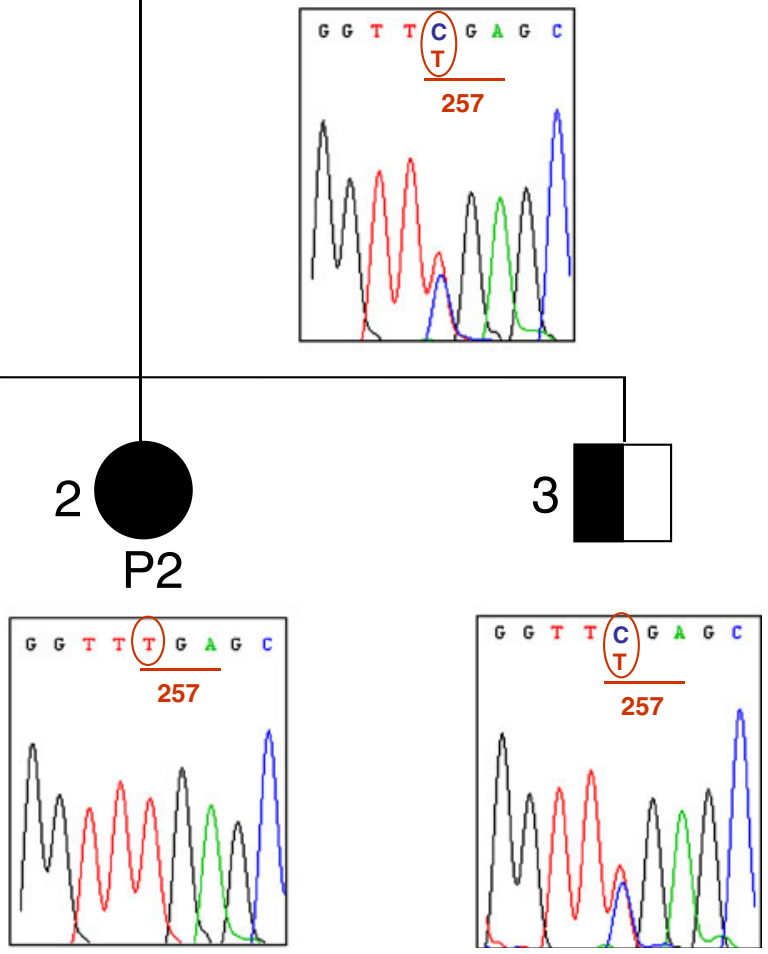

b

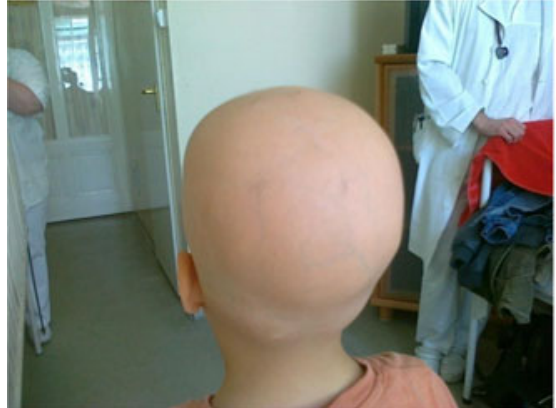

Fig. 1 Pedigrees, clinical features and AIRE mutation of APECED patients. a Pedigree and AIRE sequence variants of Patients 1 and 2. Patients with APECED (II/1 and II/2) are homozygous for the c.769C> $\mathrm{T}$ nonsense mutation predicting a stop codon in the AIRE gene, whereas the asymptomatic parents (I/1 and I/2), and sister (II/3) are heterozygous. The position of the nucleotide change in AIRE is circled and the codons affected by the mutation are underlined. $\mathbf{b}$ Alopecia totalis of Patient 1 (left) and nail candidiasis of Patient 2 (right) carrying the same c. $769 \mathrm{C}>\mathrm{T}$ homozygous nonsense mutation. $\mathbf{c}$ Pedigree and mutant AIRE sequences of Patient 3 (II/4). This patient was homozygous for the c.769C $>$ T

$11.5 \mathrm{G} / \mathrm{l})$ and granulocytosis (9.14 G/l; normal range: 1.9 $7.7 \mathrm{G} / \mathrm{l})$. IgG and IgM levels were $38.4 \mathrm{~g} / \mathrm{l}$ and $2.35 \mathrm{~g} / \mathrm{l}$, respectively, and serum protein electrophoresis excluded monoclonal gammopathy. Serological tests for Epstein-Barr virus, cytomegalovirus, hepatitis A-, B-, C-, and E virus infections were negative. Recurrent maculopapular,

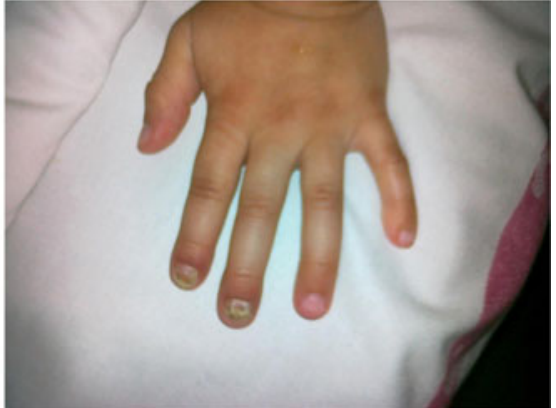

nonsense mutation of AIRE. The position of the mutated nucleotides is circled and the codons affected by the mutation are underlined. One of the sisters (II/ $/ 3$ ) of Patient 3 died of adrenal failure at the age of 10 years and no DNA sample was available for genetic testing. $\mathbf{d}$ Schematic representation of the c.769C $>\mathrm{T}$ (R257X) mutation (black squares) and a silent sequence variant (c.681C>T; p.G227G; green ovals) in the family of Patient 3 (see also c). The mother (I/2) and two children (II/1 and II/2) are heterozygous for both the pathologic and silent mutations, whereas the father is heterozygous (I/1) and Patient 3 (II/4) is homozygous for the disease-causing mutation. e Pedigree and AIRE mutation of Patient 6

morbilliform and urticarial erythema with fever have also been reported in other cases of APECED, with skin biopsy in some cases revealing lymphoplasmacytic vasculitis [38]. Liver protection therapy (silimarin, vitamin B complex) and local antifungal treatment with borax-glycerin and nystatin were initiated. Four weeks after the cessation of IVIG, serum Ig and 
C

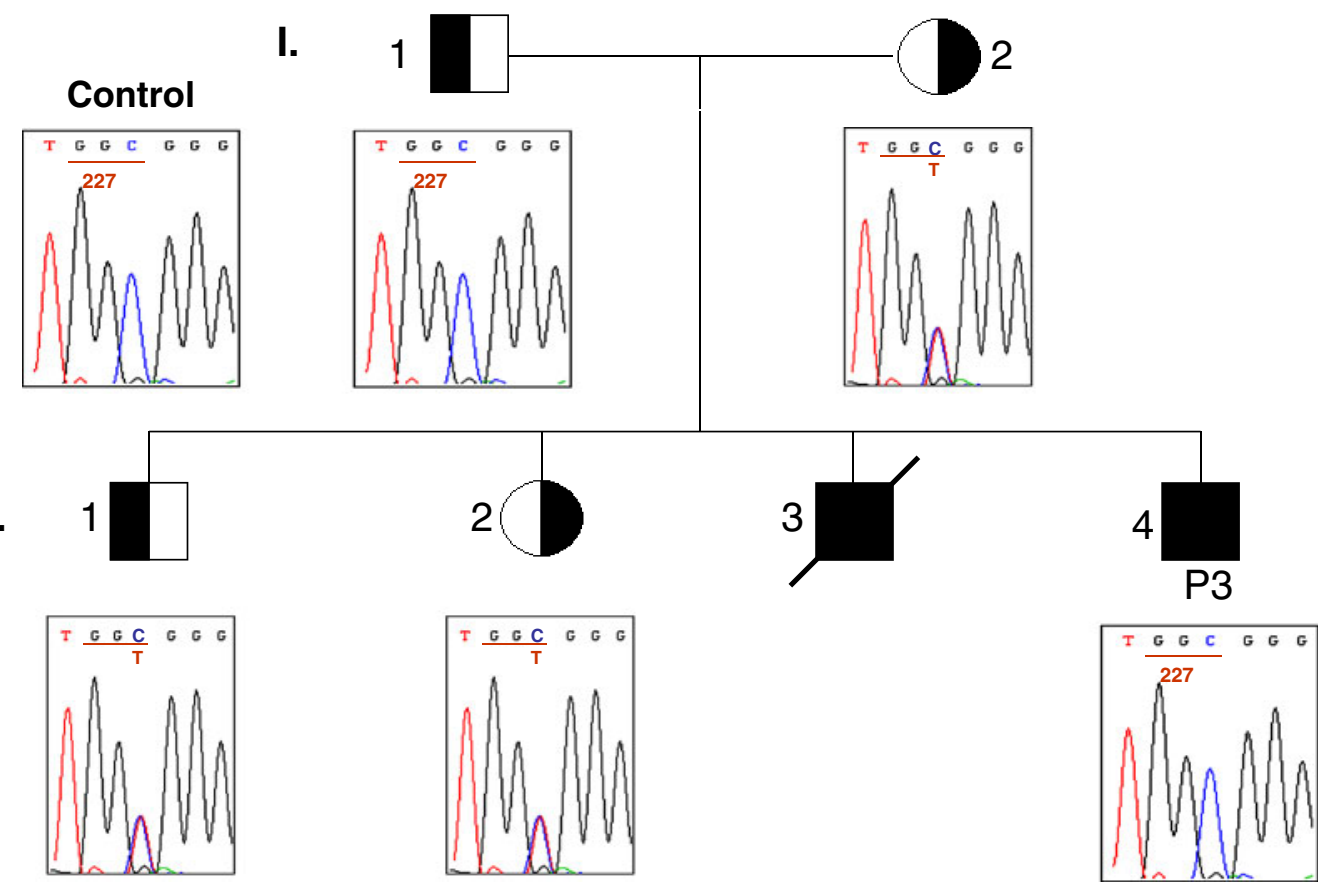

d

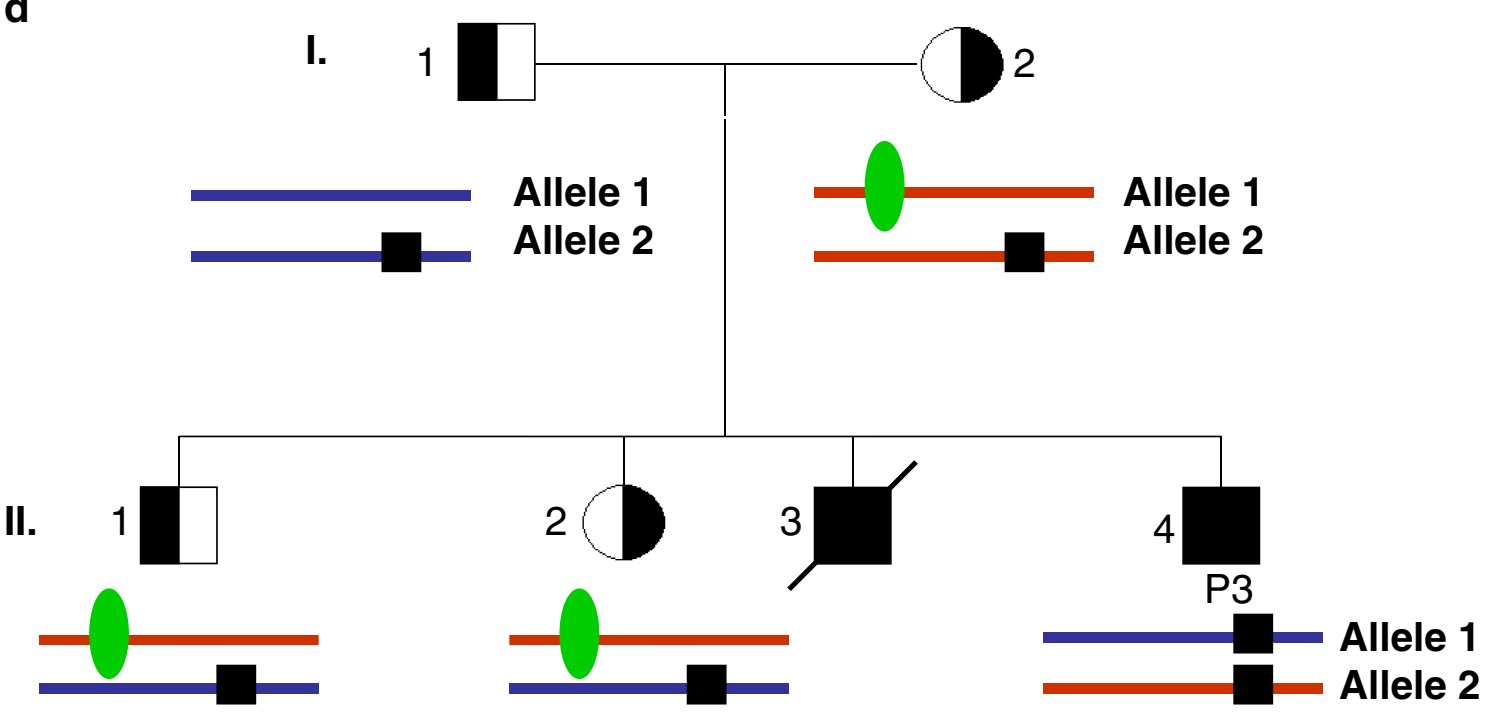

\section{Polymorphism}

Fig. 1 (continued)

liver enzyme levels gradually decreased, but the oral candidiasis remained resistant to local and oral treatment. Patients 1 and 2 were from the same family and had the same AIRE mutation, but had completely different clinical phenotypes. Patient 1 developed alopecia totalis after contracting chickenpox. He also has nail dystrophy without Candida infection. Patient 2 has been suffering from CMC since the age of 18 months. She also developed impaired liver function at the age of 4 years (Table I).

Patient 3

This 5 -year-old boy was first seen at our department at 3 weeks of age. His sister was diagnosed with APECED at 2 years of 
e

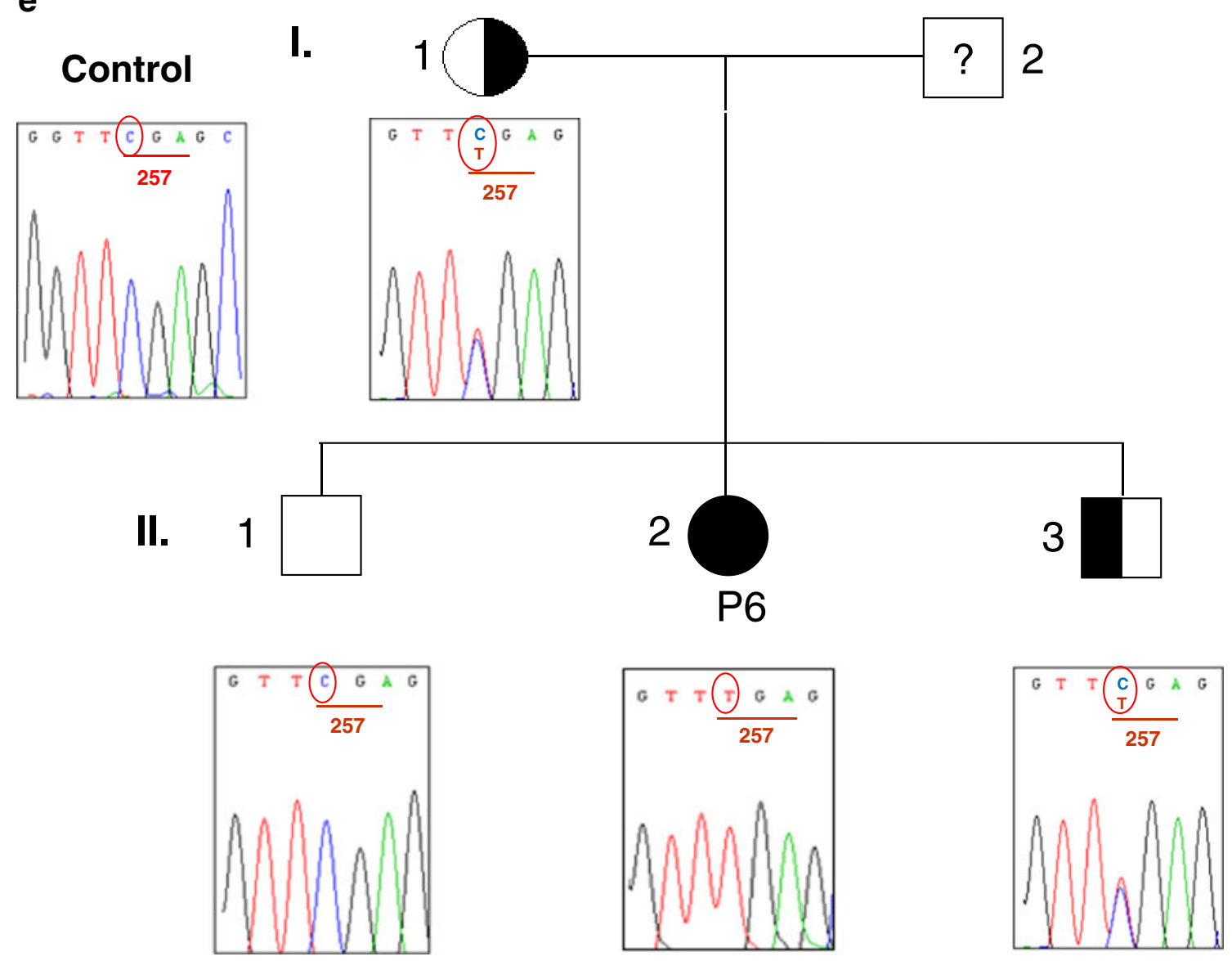

Fig. 1 (continued)

age, she had had severe CMC since early infancy, recurrent respiratory infections, adrenal and parathyroid insufficiency. She died from adrenal failure during an episode of acute bacterial infection and dehydration (Fig. 1c).

Based on the family history and in response to parental requests, we performed molecular genetic analysis and regular follow-up for this boy. From the ages of 12 to 36 months he had mild persistent hyperphosphatemia (Table I). Determinations of serum immunoglobulin isotypes showed low $\operatorname{IgA}$ and high IgG levels (Table I). Weakly positive results were obtained only once for anti-adrenal and anti-islet cell organ-specific autoantibodies. This 5 -year-old patient has no clinical signs of disease, but has produced high levels of anti-cytokine autoantibodies since infancy (Fig. 2).

Endocrine organ involvement differed considerably between these three APECED patients. Patient 1 had no endocrine organ deficiency or electrolyte imbalance, or other signs of autoimmune disease, despite the persistent detection of anti-adrenal autoantibodies. Patient 2 has hypoparathyreosis with low PTH concentration, hypocalcemia and hyperphosphatemia. She has high liver enzyme and serum IgG and M levels. Patient 3 showed subclinical signs of hypoparathyreosis, with slightly high phosphate levels and high levels of IgG. He had a transiently low cortisol concentration, with no anti-adrenal autoantibodies. The GAD antibody was detected during a transitional period.

\section{Patient 4}

This 27-year-old man had recurrent sinusitis and allergic atopic dermatitis from childhood. He developed hypoparathyroidism and hypocalcemia at the age of 9.5 years and adrenal hypofunction at 11 years of age. His parents are healthy and his only sibling (Patient 5) also has APECED. At the age of 14 years, he was diagnosed with corneal degeneration and cataracts and subsequently underwent several ophthalmological operations and corneal abrasion. He has suffered from recurrent non-purulent conjunctivitis and atopic allergic asthma. This patient has not had chronic mucosal or skin candidiasis; he presented with intermittent angular cheilitis since the age of 21 years, with no microbiological confirmation of a candidial etiology. He occasionally has palpitations and weakness, and cardiological examination revealed mitral prolapse with mild regurgitation. He has been on regular calcium and 


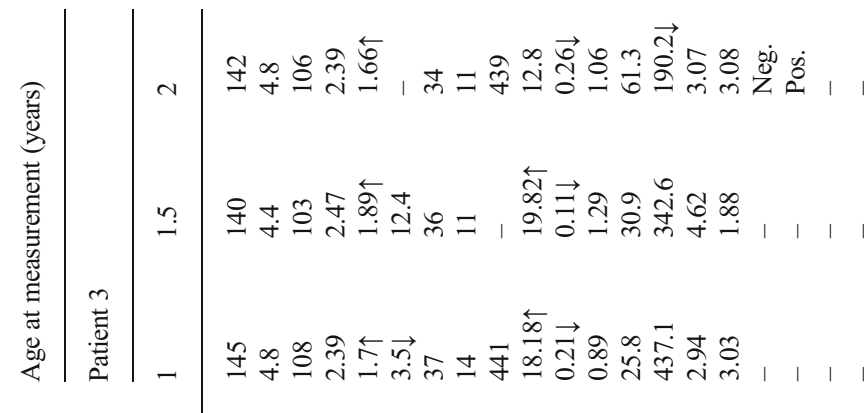

营

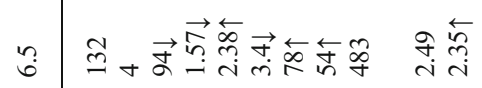

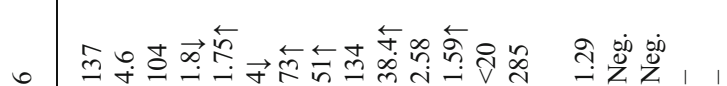

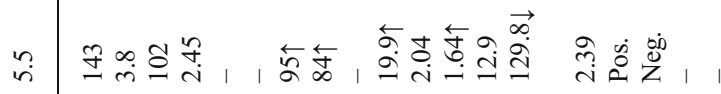

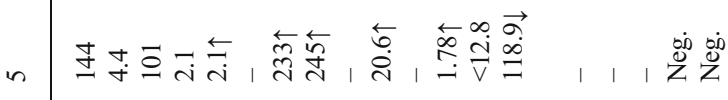

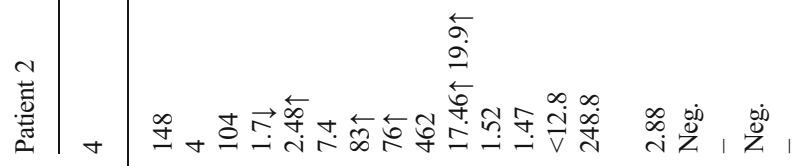

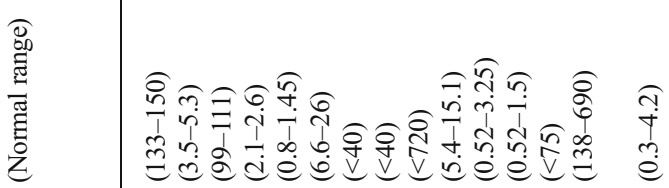

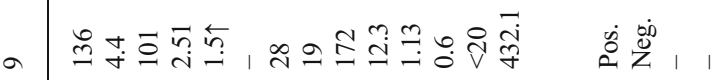

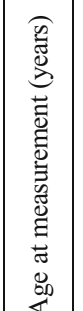

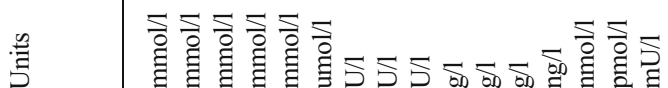

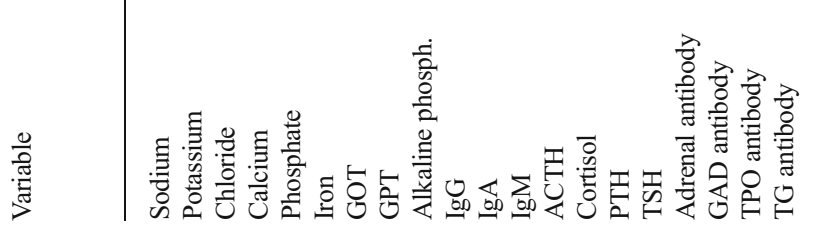

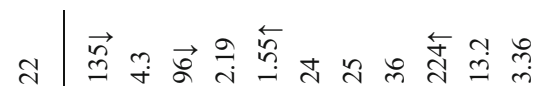

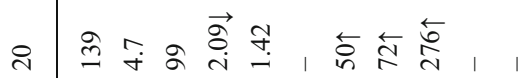

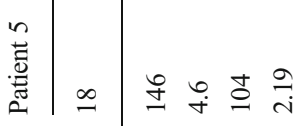

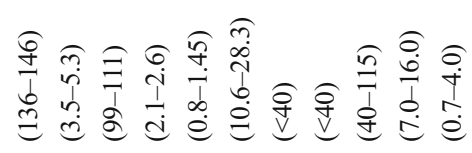

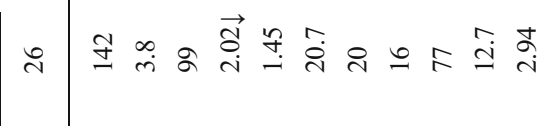

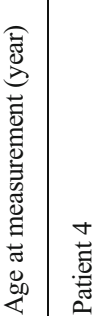

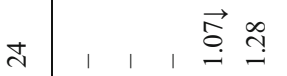

正

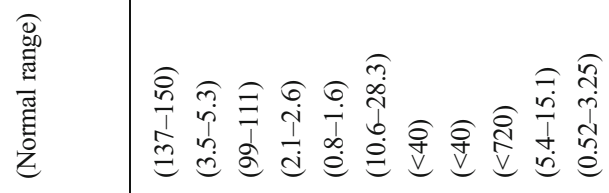

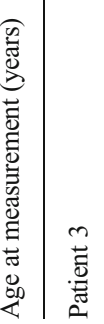

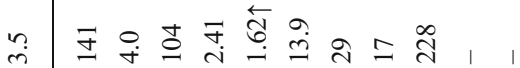

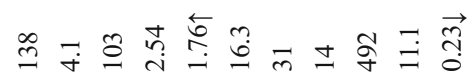

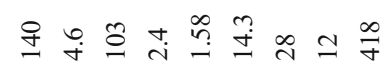

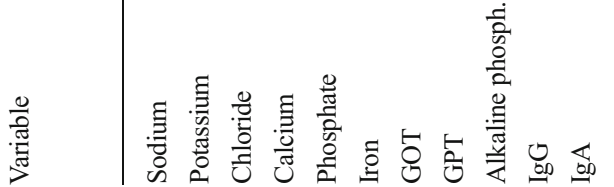


active vitamin D supplementation, with hydrocortisone replacement since the development of endocrinopathy during childhood.

\section{Patient 5}

This 22-year-old man (the brother of patient 4) presented with weakness of the extremities and muscle cramps associated with a severe episode of viral gastrointestinal infection at the age of 11 years. Chvostek's sign was positive; laboratory tests showed hypocalcemia and very low parathormone levels and the patient was diagnosed with hypoparathyreosis (Table I). At the age of 17 years, he developed isolated nail candidiasis of the right thumb. Candidial infection recurred several times at this anatomic location, but the patient has no mucosal, cutaneous or nail candidiasis at any other site. At the age of 21 years, this patient presented with persistent and progressive weight loss, and laboratory tests revealed hypoadrenia, with high levels of ACTH and lower levels of cortisol (Table I). His endocrine status has stabilized on substitution therapy.

\section{Patient 6}

This 9-year-old girl presented with recurrent upper respiratory tract infections and purulent otitis media and was frequently treated with antibiotics between the ages of 18 months and 5 years. Bilateral paracentesis was performed several times and, at the age of 5 years, a tube (grommet) was implanted, resulting in significant clinical improvement. The patient's speech and communication development and social adaptation were delayed and audiology analysis revealed mixed-type hearing impairment necessitating the use of a hearing aid. At the age of 8 years, this patient presented with muscle cramps and tetany in association with an acute episode of viral gastrointestinal infection. Laboratory tests revealed hypocalcemia and hypoparathyreosis. Until the age of 3 years, P6 presented short episodes of mild soor oris and vulvovaginitis about once per year, which have not occurred since.

\section{Analysis of AIRE Mutations}

Genomic DNA was sequenced by amplifying the exons and flanking intron regions of AIRE by PCR. Amplicons were sequenced with the Big Dye Terminator cycle sequencing kit (Applied Biosystems, Foster City, CA, USA) and analyzed with an ABI PRISM 3130 capillary sequencer (Applied Biosystems). Sequence variations were described with respect to a reference sequence (GenBank accession No. ENST00000291582) for AIRE cDNA, in which the c. 1 position corresponds to the A of the ATG translation initiation codon. Mutations are designated as recommended by den Dunnen and Antonarakis [7]. 

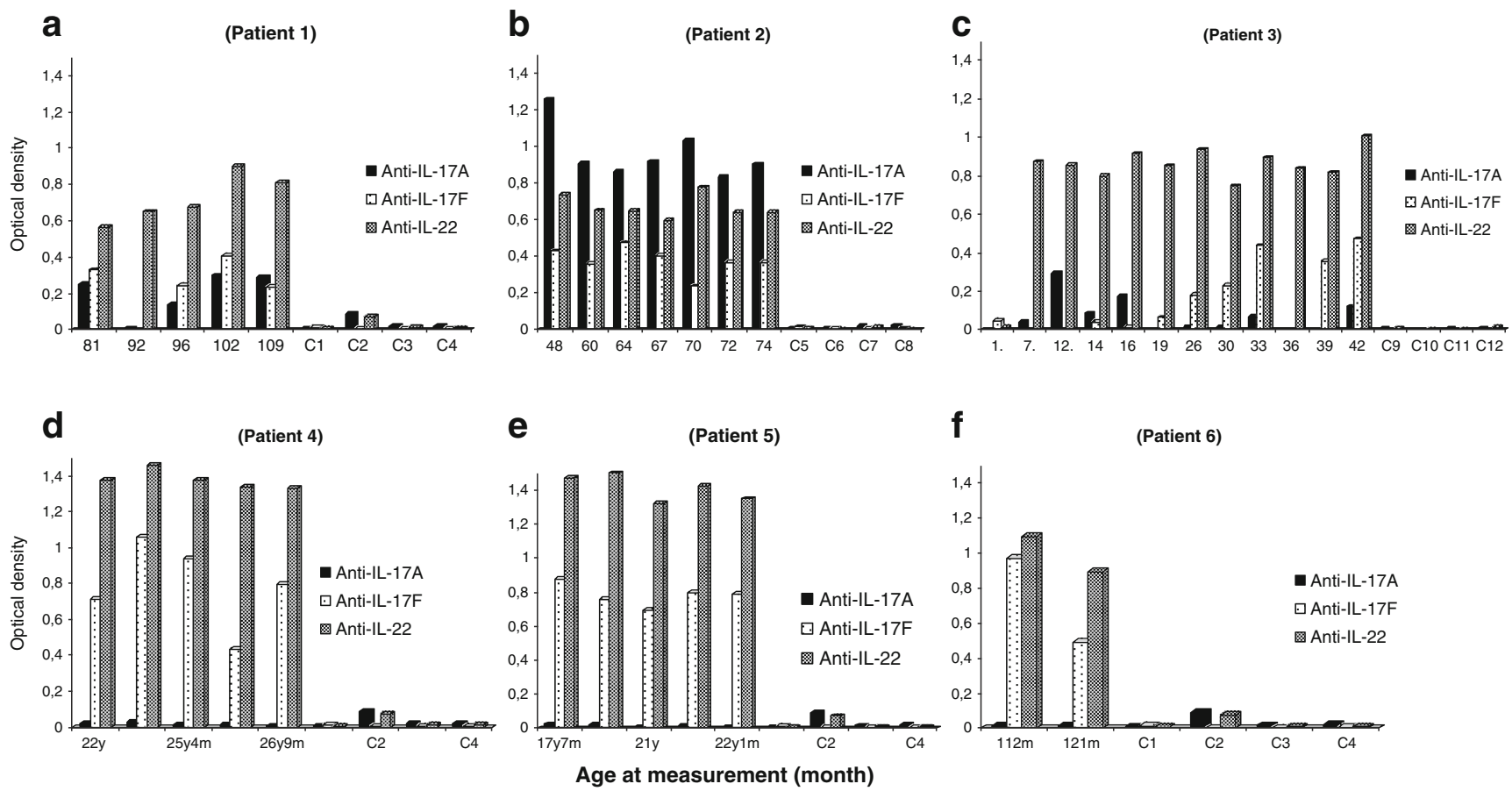

Fig. 2 Autoantibodies against IL-17A, IL-17F and IL-22 in the sera of APECED patients. In Patients $1,3,4,5$, and 6 (a and $\mathbf{d}-\mathbf{f}$ ) with no candidiasis or with mild and transient CMC, levels of anti-IL-17A autoantibodies, as measured by OD values, were low or undetectable, contrasting with the persistently high levels of anti-IL-17A antibodies in Patient 2 (b), who presented with severe and persistent CMC from the age of 18 months. Except for P2, anti-IL-22 antibody OD values were higher, comparing to antibodies to other cytokines in all patients $(\mathbf{a}-\mathbf{f})$. We

\section{Serum Samples}

Blood was taken under aseptic conditions and serum was isolated by centrifuging blood at $2,500 \mathrm{rpm}(550 \times \mathrm{g})$ for $10 \mathrm{~min}$ at room temperature. Aliquots of the samples from the patients and healthy controls were frozen at $-20^{\circ} \mathrm{C}$ until use.

\section{Routine Laboratory Assays}

Anti-endocrine organ antibody levels, clinical chemistry and immunology parameters were determined by routine laboratory assays with freshly isolated serum.

\section{Anti-Cytokine Antibody Assays}

Levels of serum autoantibodies binding to IL-17A, IL-17F, IL-22, IFN- $\alpha$ and IFN- $\omega$ were determined by ELISA. Briefly, cytokine samples (IL-17A, IL-17F and IL-22 from Peprotech, EC, London; INF- $\alpha$ and IFN- $\omega$ from Rocky Hill, NJ, USA) were diluted in PBS $(0.1 \mu \mathrm{g} / \mathrm{ml})$. We then coated Nunc MaxiSorp immunosorbent 96-well flat-bottomed plates with $100 \mu \mathrm{l}$ of cytokine suspension/well and incubated the plates overnight at $4{ }^{\circ} \mathrm{C}$. The supernatant was removed and the nonspecific binding sites were blocked by incubation for $2 \mathrm{~h}$ obtained similarly high OD values for anti-IL-17F antibodies in the sera of APS-1 patients with severe oral and therapy-resistant nail candidiasis and intermittent angular cheilitis (b), patients with mild intermittent angular cheilitis or mild, isolated nail candidiasis (d, e) and patients without candidiasis (a, c, f). OD values for anti-IL-17F autoantibodies were higher than anti-IL17-A values for the sera of all patients without severe CMC (a, c, d, e, f), but were lower in the serum of Patient 2 with severe candidiasis (b)

at room temperature with $100 \mu \mathrm{l}$ of blocking buffer $(0.1 \%$ Tween-20, $3 \%$ bovine serum albumin in PBS) per well. The plates were then washed four times with washing buffer $(0.1 \%$ Tween-20 in PBS). Serum samples were diluted 1:1000 in blocking buffer, and $100 \mu \mathrm{l}$ of the dilutions was added to wells and incubated for $1 \mathrm{~h}$ at room temperature. The plates were washed four more times. Alkaline phosphatase-conjugated goat anti-human IgG was diluted 1:10000 in antibody buffer, and $100 \mu \mathrm{l}$ was added to each well. The plates were then incubated for $1 \mathrm{~h}$ at room temperature. The plates were washed five times with washing buffer and antibody binding was visualized by adding a solution of the substrate, $p$-nitrophenol, according to the manufacturer's protocol and determining absorbance at $405 \mathrm{~nm}$, with a Multiskan EX photometer (Thermo Scientific, Shanghai, China), after incubation in the dark for $4.5 \mathrm{~min}$.

\section{Candida}

C. albicans (ATCC 10231) was maintained on Sabouraud dextrose agar at $4{ }^{\circ} \mathrm{C}$ and stationary-phase cultures were prepared by inoculating $2 \mathrm{ml}$ of DMEM. Heat-killed Candida was prepared by inoculating $5 \mathrm{ml}$ SalSol (TEVA, Debrecen, Hungary) with Candida and incubating the culture at $56^{\circ} \mathrm{C}$ for $60 \mathrm{~min}$. The Candida suspension was then centrifuged at 
$4,000 \times g$ for $10 \mathrm{~min}$ and the cell pellet was resuspended in Krebs-Ringer phosphate buffer with dextrose (KRPD). The density of heat-inactivated Candida was adjusted to $10^{6} / \mathrm{ml}$ with a McFarland densitometer.

\section{Human Blood Cells}

Peripheral blood mononuclear cells (PBMCs) were isolated from the patients and controls. After density gradient centrifugation and removal of the mononuclear cell layer, the cells were washed several times in KRPD. The cells were resuspended in Dulbecco's modified Eagle's Medium (DMEM; Sigma-Aldrich, St. Louis, MO). PBMCs $\left(5 \times 10^{5}\right.$ cells/well $)$ were incubated either alone or in the presence of heat-killed C. albicans, at $37{ }^{\circ} \mathrm{C}$, in 96 -well round-bottomed culture plates. After 5 days of incubation, the plates were centrifuged and the supernatants were removed and stored at $-20{ }^{\circ} \mathrm{C}$ for the determination of cytokine concentrations.

\section{Measurement of Cytokine Concentrations}

The concentrations of IL-17A, IL-17F, IL-22 and TNF- $\alpha$ in the supernatants of Candida-exposed PBMCs were determined by sandwich enzyme-linked immunosorbent assays (Quantikine, R\&D Systems, MN, USA). All experiments were performed in duplicate or triplicate.

\section{Results}

Genetic Data and Clinical Presentation

Four patients were found to be homozygous for the c. $769 \mathrm{C}>\mathrm{T}$ nonsense mutation of the AIRE gene (Fig. 1a, c, and e), which replaces the arginine codon in position 257 with a stop codon (R257X/R257X). The parents of Patients P1, P2, and P3 and the mother of Patient 6 were heterozygous for the mutant allele, consistent with autosomal recessive inheritance for this trait. Heterozygosity was also detected in a male sibling of Patients 1 and 2 (Fig. 1a), in a brother and a sister of Patient 3 (Fig. 1c), and in one of the two brothers of Patient 6. Genomic DNA sequencing of samples from the mother and two siblings of Patient 3 revealed a silent mutation in one allele of AIRE in addition to the disease-causing mutation located in the other allele (Fig. 1d).

Patients P4 and P5 carry the c.769C > T/c.1344delC compound heterozygous mutation in the AIRE gene, as described in detail elsewhere [36].

Autoantibodies Against IL-17A, IL-17F and IL-22

OD values for autoantibodies against IL-22 were higher for all patients than for healthy controls (Fig. 2). In Patient 1, anti-IL-
22 values increased gradually between the ages of 81 and 102 months (Fig. 2a). OD values for anti-IL-22 antibodies were slightly higher in the adult patients (Fig. $2 \mathrm{~d}$ and e) than in children. By contrast, the levels of anti-IL-17A autoantibodies differed considerably between patients. These levels were higher in Patient 2 than in the other patients. Patient 2, who had presented CMC since the age of 18 months, had persistently high anti-IL-17A levels (Fig. 2b). Patient 2 was referred to our center at the age of 4 years, and no serum samples were available for antibody testing before this age. Her brother, who had no signs of CMC (Patient 1), had low or undetectable levels of anti-IL-17A antibodies over the period of observation. Patient 3, with no signs of mucosal or skin candidiasis, also had low or undetectable anti-IL-17A antibody levels over a period of 4 years. Similarly, OD values for anti-IL-17A antibodies in Patients P4, P5, and P6, who had candidiasis of a single fingernail beginning at the age of 17 years (P5), angular cheilitis of unknown etiology since the age of 21 years (P6) or three short episodes of mucosal candidial disease during the first 3 years of life (P6), were negligible, as shown by comparison with control values and with those for Patient 2, who had prolonged, severe CMC (Fig. 2b, d-f). OD values for IL-17F were intermediate, between those obtained for antiIL-17A and anti-IL-22 antibodies, and were not correlated with the severity of CMC (Fig. 2a-f).

Experiments were performed to measure OD values of anti-IL-17A and anti-IL-22 in sera of Patients 1, 2 and 3 by using varying serum concentrations (Fig. 3). Data of these experiments confirmed the differences in OD values found with a single dilution of sera (Fig. 2).

\section{Autoantibodies Against Type I Interferons}

OD values for antibodies against IFN- $\alpha$ and IFN- $\omega$ were measured in three patients, and were high and similar in Patients 1 and 3 (Fig. 4). In Patient 2, with CMC, the low anti IFN- $\omega$ antibody level contrasted with the high level of antiIFN- $\alpha$ antibodies (Fig. 4b). In Patient 3, who was followed up from early infancy, we observed a gradual increase in the levels of IFN- $\alpha$ and IFN- $\omega$ antibodies until the age of 14 months (Fig. 4c). Autoantibody production began at the age of 7 months and sustained increases in the levels of these antibodies resulted in high OD values later on.

\section{Release of Cytokines by Candida-Exposed Blood Cells}

We measured the release of IL-17A, IL-17F, and IL-22 by PBMCs after 5 days of stimulation with heat-killed Candida (Fig. 5). PBMCs from patients released negligible or small amounts of anti-cytokine antibodies on exposure to Canadida, compared to cells from healthy controls (Fig. 5). These data suggest an impaired 'Th-17-type' response to Candida in patients with APECED. To prove the possibility of a broader 

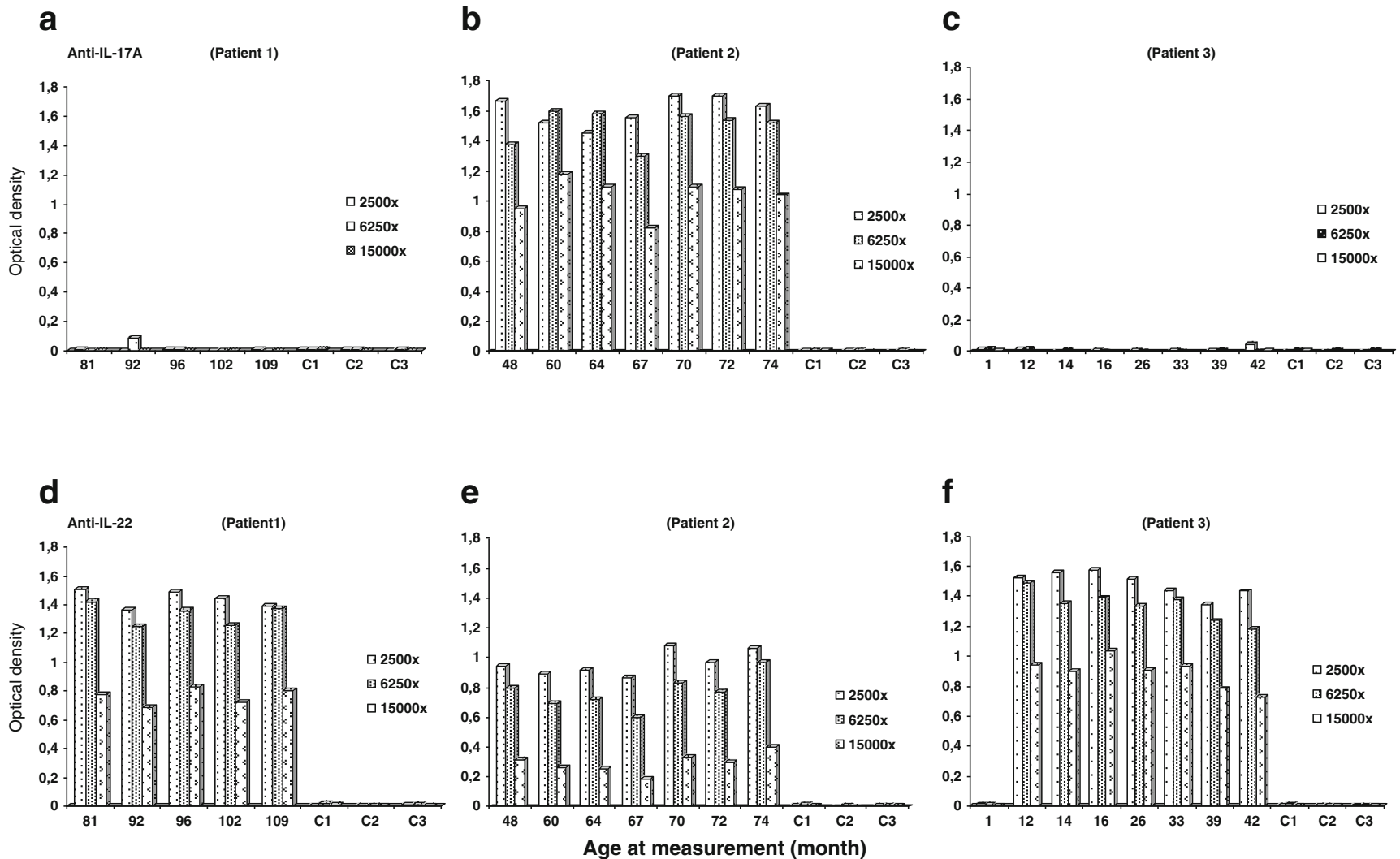

Fig. 3 Anti-IL-17A and anti-IL-22 OD values in different dilutions of serum samples. High OD values for anti-IL-17A antibodies were found in each dilutions of sera from Patient 2 (P2), compared to values found in

defect of cytokine release by Candida-exposed PBMCs, we measured concentrations of TNF- $\alpha$ in supernatants as positive control. The data of these control experiments showed that release of TNF- $\alpha$ by cells from patients and controls were comparable (Fig. 5).

\section{Discussion}

The importance of the IL-17 family of cytokines in immunity to Candida is well established [5, 15, 19, 29, 35]. Previous sera of $\mathrm{P} 1$ and $\mathrm{P} 3$. In contrast, OD values of anti-IL-22 antibodies were high in all patients with somewhat lower values found in P2 compared to those detected in $\mathrm{P} 1$ and $\mathrm{P} 3$

studies have suggested that anti-cytokine autoantibodies are involved in CMC pathogenesis in APECED patients [11, 27, $28,36]$. It has been suggested that autoantibodies against cytokines, including IL-17A, IL-17F and IL-22, may underlie CMC in patients with APECED [11, 28]. Recent findings have suggested that the presence of autoantibodies against IL-22 may be a useful predictor of CMC [11, 12]. It has also been suggested that IL-17 plays a key role in protection against Candida infection, and some patients with isolated CMC have smaller than normal proportions of IL-17-producing $\mathrm{T}$ cells, and produce only low levels of IL-17 [15, 27, 29].
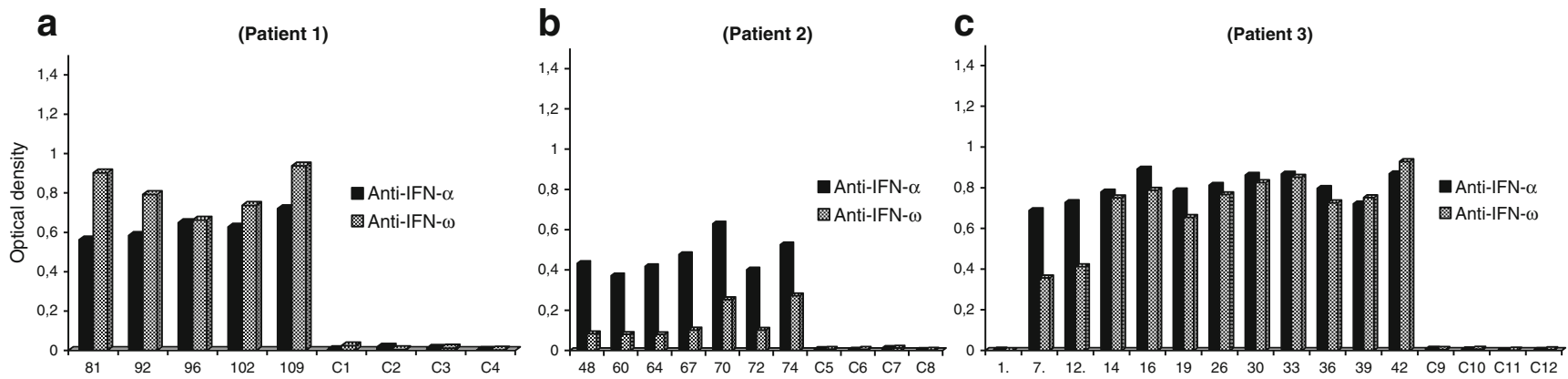

Age of mesurement (months)

Fig. 4 Autoantibodies against IFN- $\alpha$ and IFN- $\omega$ in the sera of APECED patients. In Patients 1 and 3 (a and $\mathbf{c}$ ), the OD values for both anti-IFN- $\alpha$ and antiIFN- $\omega$ antibodies were persistently high. Intriguingly, serum OD values for anti-IFN- $\alpha$ and anti-IFN- $\omega$ antibodies were relatively low in Patient 2 (b) 


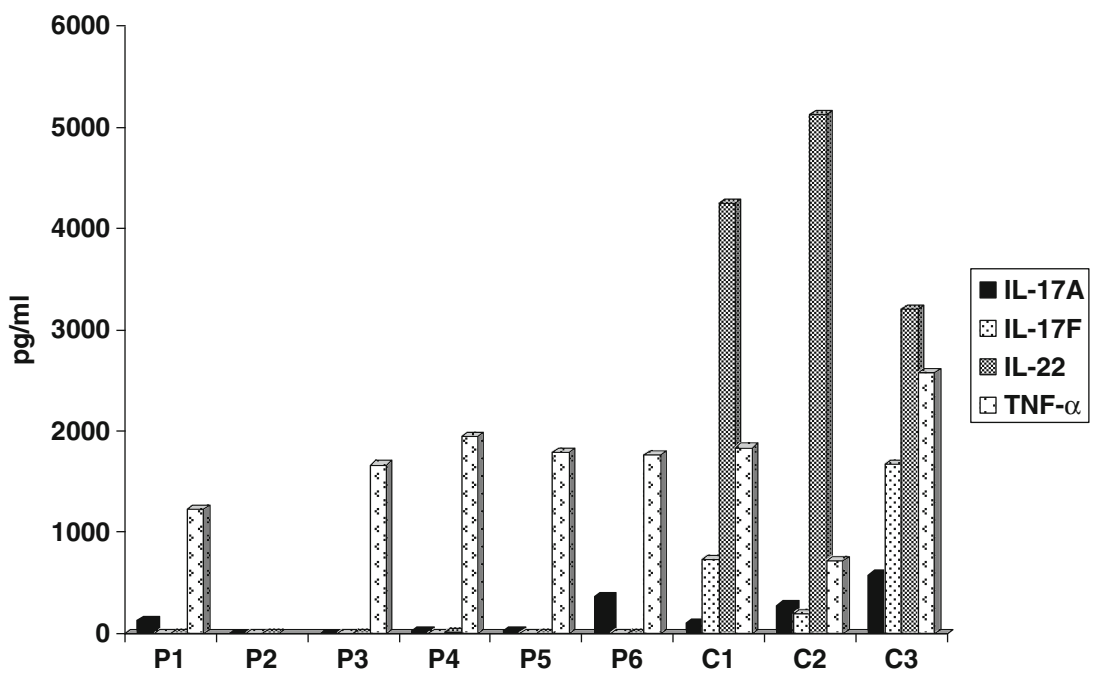

Fig. 5 IL-17A, IL-17F, and IL-22 secretion by blood cells exposed to Candida. Peripheral blood mononuclear cells were stimulated with heatkilled Candida blastoconidia for 5 days at $37^{\circ} \mathrm{C}$. Cells were then centrifuged and cytokine concentration was measured in the supernatants by
ELISA, as described in "Methods". We found impaired secretion of IL17A, IL-17F, and IL-22 by cells from APECED patients with or without severe candidiasis, as shown as compared with cells from healthy controls. In contrast, the release of TNF- $\alpha$ was comparable in patients and controls
In this study, Patient 2, with severe and prolonged CMC, presented high levels of autoantibodies against IL-17A. The other five patients with the same genotype or heterozygous AIRE mutation had low or undetectable anti-IL-17A antibody levels and were asymptomatic or presented only mild and transient signs of mucocutaneous candidiasis. Previous studies have suggested that IL-22 plays an important role in the protection of skin and epithelial surfaces against Candida infection [6, 39]. It has been reported that IL-22-producing cells help to protect mucosal surfaces against fungal infection in conditions of defective adaptive immunity, through a primitive antifungal effector mechanism [6, 39]. Furthermore, IL22 has been reported to play a role in candidiasis, by controlling fungal invasion and epithelial homeostasis [39]. All six patients included in this study had high levels of anti-IL-22 autoantibodies, but only Patient 2, with high levels of anti-IL17A antibodies, displayed a predisposition to severe CMC. Furthermore, OD values for anti-IL-17F antibodies were high in all patients, but those of Patient 2 were no higher than those in the other patients. These findings argue against a primary role of anti-IL-17F and anti-IL-22 antibodies in the susceptibility of APECED patients to CMC, instead favoring the concept that anti-IL17A antibodies are the primary components of impaired anti-candidial immunity in APECED patients. Measuring functional activity of these antibodies could have confirmed further our findings but such assays are not currently available in our lab.

The role of anti-IFN antibodies in the pathogenesis of disease signs in APECED patients is not fully understood. Recent studies have shown that high levels of autoantibodies against type I IFNs, including IFN- $\alpha$ and IFN- $\omega$ in particular, are found in most patients with APECED, suggesting that
anti-IFN autoantibodies are hallmarks of the disease [10, 25]. These studies showed that autoantibodies against IFN- $\omega$ were clearly present in all patients and that their levels were not clearly correlated with the number or duration of disease manifestations [17]. We found high levels of autoantibodies against IFN- $\alpha$ in the sera of three patients studied, and of autoantibodies against IFN- $\omega$ in Patients 1 and 3. The level of anti-IFN- $\omega$ autoantibodies was low in Patient 2. These data do not support the existence of a relationship between APECED severity and anti-IFN autoantibody level. The role of the variable levels of anti-IFN- $\omega$ antibodies in this multiorgan primary immunodeficiency disorder therefore remains to be elucidated.

Recent studies suggested that impairment of IL-17F and IL-22 secretion by PBMC's of APECED patients may be an important precipitating factor in predisposition to candidiasis $[11,23]$. The role of IL-17A secretion seems somewhat controversial in these studies. One of them found increased numbers of C. albicans-induced IL-17A-producing cells in PBMC from APECED patients, measured by flow cytometry by intracellular staining of IL-17A [1], in contrast with other studies, which consistently found impaired IL-17A responses in APECED patients [8, 11, 23]. PBMCs from our patients released small amounts of IL-17A and much smaller, or negligible amounts of IL-17F and IL-22 by Candidaexposed cells, compared with healthy controls. We found impaired secretion of IL-17A, IL-17F and IL-22 by PBMC cells from APECED patients, with, or without severe candidiasis, in contrast the release of TNF- $\alpha$, which was comparable with healthy controls.

We provide the first description of the possible effects of IVIG for treating severe CMC in APECED patients, and the 
transient improvement of mucosal candidiasis following monthly IVIG infusions in Patient 2 was intriguing. IVIG replacement therapy is widely used to prevent infectious complications in patients with primary immunodeficiency disorders $[13,24]$. In addition to prophylaxis, IVIG concentrates may also be helpful for the treatment of various inflammatory conditions [21, 33]. However, the mode of action of this therapy in autoimmune diseases remains poorly understood. IVIG contains anti-idiotype antibodies against diseaseassociated autoantibodies [21]. These anti-idiotype antibodies may be able to bind and neutralize the pathogenic anticytokine antibodies typically produced by patients with APECED, consistent with the decrease in autoantibody levels observed in other conditions following IVIG therapy [32]. We believe that IVIG treatment is unlikely to have increased the efficiency of Candida elimination by opsonophagocytosis, given the clinical observation that patients with X-linked agammaglobulinemia characterized by an almost complete lack of serum immunoglobulins typically do not suffer from CMC or invasive candidial disease [14]. We observed no detectable decrease in anti-IL-17A and anti-IL-22 antibody levels IVIG therapy in Patient 2 (data not shown), but we believe that further studies of possible therapeutic effects of IVIG in APECED patients with severe $\mathrm{CMC}$ are warranted, based on our observation.

\section{Conclusion}

We report here a correlation between anti-IL-17A autoantibody level and predisposition to chronic mucocutaneous candidiasis in APECED patients. We suggest that anti-IL-17A autoantibodies may play a more important role than anti-IL-22 and anti-IL-17F antibodies in rendering APECED patients susceptible to candidiasis. We suggest that high levels of anti-IL-22 and anti-IL-17F autoantibodies may not be correlated with the occurrence of candidiasis in APECED patients. We present data suggesting that the release of IL-17A, IL-17F and IL-22 by blood cells is impaired in APECED patients. These data suggest that autoantibodies against cytokines may only partly account for the susceptibility to Candida in APECED patients. Other mechanisms may also be involved. We suggest that IVIG substitution may be of use in APECED patients with $\mathrm{CMC}$, to lower the burden and complications of candidiasis on body surfaces.

Acknowledgments This work was supported by the UD Faculty of Medicine Research Fund (Bridging Fund 2012) and the TÁMOP 4.2.2.A-11/1/KONV-2012-0023 "VÉD-ELEM" project grant to LM. We thank Drs. A. B. Wolff, É. Oláh and the members of our laboratory team for helpful discussion, and to Drs. L. Gajdos, Zs. Petrekanits, and J. Boda for referring the patients.

Conflict of interest statement The authors have no conflicts of interest.

\section{References}

1. Ahlgren KM, Moretti S, Lundgren BA, Karlsson I, Ahlin E, Norling A, et al. Increased IL-17A secretion in response to Candida albicans in autoimmune polyendocrine syndrome type 1 and its animal model. Eur J Immunol. 2011;41:235-45.

2. Ahonen P, Myllarniemi S, Sipila I, Perheentupa J. Clinical variation of autoimmune polyendocrinopathy-candidiasis-ectodermal dystrophy (APECED) in a series of 68 patients. N Engl J Med. 1990;322: 1829-36.

3. Al-Herbish AS, Bailey JD, Kooh SW. Growth hormone deficiency in autoimmune polyglandular disease type 1. Saudi Med J. 2000;21(8): $765-8$.

4. Collins SM, Dominguez M, Ilmarinen T, Costigan C, Irvine AD. Dermatological manifestations of autoimmune polyendocrinopathycandidiasis-ectoderma dystrophy syndrome. Br J Dermatol. 2006;154(6):1088-93.

5. Cypowyj S, Picard C, Maródi L, Casanova JL, Puel A. Immunity to infection in IL-17-deficient mice and humans. Eur J Immunol. 2012;42(9):2246-54.

6. De Luca A, Zelante T, D'Angelo C, Zagarella S, Fallarino F, Spreca A, et al. IL-22 defines a novel immune pathway of antifungal resistance. Mucosal Immunol. 2010;3(4):361-73.

7. den Dunnen JT, Antonarakis E. Nomenclature for the description of human sequence variations. Hum Genet. 2001;109:121-4.

8. Eyerich K, Foerster S, Rombold S, Seidl HP, Behrendt H, Hofmann $\mathrm{H}$, et al. Patients with chronic mucocutaneous candidiasis exhibit reduced production of Th17-associated cytokines IL-17 and IL-22. J Invest Dermatol. 2008;128(11):2640-5.

9. Gupta AN, Kaniyoor Nagri S. Schmidt's syndrome - case report. Australas Med J. 2012;5(6):292-5.

10. Husebye ES, Perheentupa J, Rautemaa R, Kampe O. Clinical manifestations and management of patients with autoimmune polyendocrine syndrome type I. J Intern Med. 2009;265(5):514-29.

11. Kisand K, Boe Wolff AS, Podkrajsek KT, Tserel L, Link M, Kisand $\mathrm{KV}$, et al. Chronic mucocutaneous candidiasis in APECED or thymoma patients correlates with autoimmunity to Th17-associated cytokines. J Exp Med. 2010;207(2):299-308.

12. Kisand K, Lilic D, Casanova JL, Peterson P, Meager A, Willcox N. Mucocutaneous candidiasis and autoimmunity against cytokines in APECED and thymoma patients: clinical and pathogenetic implications. Eur J Immunol. 2011;41:1517-27.

13. Kreuz W, Erdös M, Rossi P, Bernatowska E, Espanol T, Maródi L. A multi-centre study of efficacy and safety of intratect, a novel intravenous immunoglobulin preparation. Clin Exp Immunol. 2010;161(3): $512-7$.

14. Maródi L. Local and systemic host defense mechanisms against Candida: immunopathology of candidal infections. Pediatr Infect Dis J. 1997;16(8):795-801.

15. Maródi L, Cypowyj S, Tóth B, Chernyshova L, Puel A, Casanova JL. Molecular mechanisms of mucocutaneous immunity against $C$ andida and Staphylococcus species. J Allergy Clin Immunol. 2012;130(5): 1019-27.

16. McManus BA, McGovern E, Moran GP, Healy CM, Nunn J, Fleming $\mathrm{P}$, et al. Microbiological screening of Irish patients with autoimmune polyendocrinopathy-candidiasis-ectodermal dystrophy reveals persistence of Candida albicans strains, gradual reduction in susceptibility to azoles, and incidences of clinical signs of oral candidiasis without culture evidence. J Clin Microbiol. 2011;49(5): 1879-89.

17. Meager A, Visvalingam K, Peterson P, Möll K, Murumagi A, Krohn $\mathrm{K}$, et al. Anti-interferon autoantibodies in autoimmune polyendocrinopathy syndrome type 1. PLoS Med. 2006;3(7):e289.

18. Meloni A, Furcas M, Cetani F, Marcocci C, Falorni A, Perniola R, et al. Autoantibodies against type I interferons as an additional 
diagnostic criterion for autoimmune polyendocrine syndrome type I. J Clin Endocrinol Metab. 2008;93(11):4389-97.

19. Meloni A, Willcox N, Meager A, Atzeni M, Wolff SA, Husebye ES, et al. Autoimmune polyendocrine syndroma type 1: an extensive longitudinal study in Sardinian patients. J Clin Endocrinol Metab. 2012;97(4):1114-24.

20. Myhre AG, Halonen M, Eskelin P, Ekwall O, Hedstrand H, Rorsman $\mathrm{F}$, et al. Autoimmune polyendocrine syndrome type 1 (APS I) in Norway. Clin Endocrinol. 2001;54:211-7.

21. Negi VS, Elluru S, Sibéril S, Graff-Dubois S, Mouthon L, Kazatchkine MD, et al. Intravenous immunoglobulin: an update on the clinical use and mechanisms of action. J Clin Immunol. 2007;27(3):233-45.

22. Neufeld M, Maclaren NK, Blizzard RM. Two types of autoimmune Addison's disease with different polyglandular autoimmune (PGA) syndromes. Medicine (Baltimore). 1981;60(5):355-62.

23. Ng WF, von Delwig A, Carmicheal AJ, Arkwright PD, Abinun M, Cant $\mathrm{AJ}$, et al. Impaired $\mathrm{T}_{\mathrm{H}} 17$ responses in patients with chronic mucocutaneous candidiasis with and without autoimmune polyendocrinopathy-candidiasis-ectodermal dystrophy. J Allergy Clin Immunol. 2010;126(5):1006-15.

24. Orange JS, Hossny EM, Weiler CR, Ballow M, Berger M, Bonilla FA, et al. Use of intravenous immunoglobulin in human disease: a review of evidence by members of the Primary Immunodeficiency Committee of the American Academy of Allergy, Asthma and Immunology. J Allergy Clin Immunol. 2006;117(4 Suppl):S525-53.

25. Perniola R, Falorni A, Clemente MG, Forini F, Accoqli E, Lobreqlio G. Organ-specific and non-organ-specific autoantibodies in children and young adults with autoimmune polyendocrinopathy-candidiasisectodermal dytrophy (APECED). Eur J Endocrinol. 2000;143(4): 497-503.

26. Peterson P, Pitkanen J, Sillanpaa N, Krohn K. Autoimmune polyendocrinopathy candidiasis ectodermal dystrophy: a model disease to study molecular aspects of endocrine autoimmunity. Clin Exp Immunol. 2004;135:348-57.

27. Puel A, Cypowyj S, Maródi L, Abel L, Picard C, Casanova JL. Inborn errors of human IL-17 immunity underlie chronic mucocutaneous candidiasis. Curr Opin Allergy Clin Immunol. 2012;12(6):616-22.
28. Puel A, Döffinger R, Natividad A, Chrabieh M, Barcenas-Morales G, Picard C, et al. Autoantibodies against IL-17A, IL-17F, and IL-22 in patients with chronic mucocutaneous candidiasis and autoimmune polyendocrine syndrome type I. J Exp Med. 2010;207(2):291-7.

29. Puel A, Picard C, Cypowyj S, Lilic D, Abel L, Casanova JL. Inborn errors of mucocutaneous immunity to Candida albicans in humans: a role for IL-17 cytokines? Curr Opin Immunol. 2010;22(4):467-74.

30. Rautemaa R, Hietanen J, Nissalo S, Pirinen S, Perheentupa J. Oral and oesophageal squamous cell carcinoma - a complication or a component of autoimmune polyendocrinopathy-candidiasisectodermal dystrophy (APECED, APS-I). Oral Oncol. 2007;43: 607-13.

31. Rosa DD, Pasqualotto AC, Denning DW. Chronic mucocutaneous candidiasis and eosophageal cancer. Med Mycol. 2008;46(1):85-91.

32. Sami N, Bhol KC, Ahmed AR. Influence of IVIg therapy on autoantibody titer to desmoglein 1 in patients with pemphigus foliaceus. Clin Immunol. 2002;105(2):192-8.

33. Sibéril S, Elluru S, Graff-Dubois S, Negi VS, Delignat S, Mouthon L, et al. Intravenous immunoglobulins in autoimmune and inflammatory disease. Ann N Y Acad Sci. 2007;1110:497-506.

34. Thorpe ES, Handley HE. Chronic tetany and chronic mycelial stomatitis in a child aged four and one-half years. Arch Pediatr Adolesc Med. 1929;38(2):328-38.

35. Tóth B, Méhes L, Taskó S, Szalai Z, Tulassay Z, Cypowyj S, et al. Herpes in STAT1 gain-of-function mutation. Lancet. 2012;379(9835): 2500.

36. Tóth B, Wolff AS, Halász Z, Tar A, Szüts P, Illyés I, et al. Novel sequence variation of AIRE and detection of interferon-omega antibodies in early infancy. Clin Endocrinol (Oxf). 2010;72(5):641-7.

37. Uittamo J, Siikala E, Kaihovaara P, Salaspuro M, Rautemaa R. Chronic candidosis and oral cancer in APECED-patients: production of carcinogenic acetaldehyde from glucose and ethanol by Candida albicans. Int J Cancer. 2009;124:754-6.

38. Weiler FG, Dias-da-Silva MR, Lazaretti-Castro M. Autoimmune polyendocrine syndrome type 1: case report and review of literature. Arg Bras Endocrinol Metabol. 2012;56(1):54-66.

39. Zelante T, Iannitti R, De Luca A, Romani L. IL-22 in antifungal immunity. Eur J Immunol. 2011;41(2):270-5. 